\title{
Topologically invariant transformation for lattice fermions
}

\author{
Ting-Wai Chiu \\ Department of Physics, National Taiwan University, Taipei 106, Taiwan, ROC
}

Received 27 January 2000; received in revised form 6 June 2000; accepted 30 June 2000

Editor: M. Cvetič

\begin{abstract}
A transformation is devised to convert any lattice Dirac fermion operator into a Ginsparg-Wilson Dirac fermion operator. For the standard Wilson-Dirac lattice fermion operator, the transformed new operator is local, free of $O(a)$ lattice artifacts, has correct axial anomaly in the trivial sector, and is not plagued by the notorious problems (e.g., additive mass renormalization) which occur to the standard Wilson-Dirac lattice fermion operator. (C) 2000 Elsevier Science B.V. All rights reserved.
\end{abstract}

\section{Introduction}

To formulate chiral fermions on the lattice, one must take into account the constraints imposed by the Nielson-Ninomiya no-go theorem [1]. It asserts that any lattice Dirac operator $D$ in the free fermion limit must violate at least one of the four basic properties [(i)-(iv) listed below] of massless Dirac fermion in continuum. Nevertheless, we can incorporate the gauge interactions in the no-go theorem as follows. Since the gauge link variables are trivial in the Dirac space, the chiral symmetry of $D$ is not affected by turning on a background gauge field. In other words, if $\left\{D, \gamma_{5}\right\}$ is zero in the free fermion limit, then it remains to be zero even when the gauge links are different from the identity. Moreover, the presence of gauge interactions does not improve the locality of $D$. Therefore, we can assert that any

E-mail address: twchiu@phys.ntu.edu.tw (T.-W. Chiu). gauge covariant Dirac operator $D$ on a finite lattice must violate at least one of the following properties:

(i) $\quad D$ is chirally symmetric. $\left(D \gamma_{5}+\gamma_{5} D=\right.$ 0$.

(ii) $\quad D$ is local. $(|D(x, y)| \sim \exp (-|x-y| / l)$ with $l \sim a$; or $D(x, y)=0$ for $|x-y|>z$, where $z$ is much less than the size of the lattice.)

(iii) In the free fermion limit, $D$ is free of species doublings. (The free fermion propagator $D^{-1}(p)$ has only one simple pole at the origin $p=0$ in the Brillouin zone.)

(iv) In the free fermion limit, $D$ has correct continuum behavior. (In the limit $a \rightarrow 0$, $D(p) \sim i \gamma_{\mu} p_{\mu}$ around $p=0$.)

During the last two years, it has become clear that the proper way to circumvent the no-go theorem is to break the chiral symmetry of $D$ (i) at finite lattice 
spacing, according to the Ginsparg-Wilson relation [2]

$$
D \gamma_{5}(\mathbb{1}-a R D)+(\mathbb{1}-a D R) \gamma_{5} D=0,
$$

where $R$ is any positive definite Hermitian operator which is local in the position space and commutes with $\gamma_{5}$. However, the GW relation should be regarded as a generalized chiral symmetry which contains the usual chiral symmetry (i) in the continuum limit $a \rightarrow 0$.

Since the massless Dirac operator in continuum is chirally symmetric $\left(\mathscr{D} \gamma_{5}+\gamma_{5} \mathscr{D}=0\right)$ and antihermitian $\left(\mathscr{D}^{\dagger}=-\mathscr{D}\right)$, so it is $\gamma_{5}$-Hermitian $\left(\mathscr{D}^{\dagger}=\right.$ $\left.\gamma_{5} \mathscr{D} \gamma_{5}\right)$. Thus, we require that the lattice Dirac operator $D$ also preserves this symmetry at any lattice spacing, i.e.,

$D^{\dagger}=\gamma_{5} D \gamma_{5}$.

The first explicit and physical $D$ satisfying the GW relation (1) (with $R=1 / 2$ ) and $\gamma_{5}$-Hermiticity is the Overlap Dirac operator $D=a^{-1}(\mathbb{1}+$ $\left.\gamma_{5} \epsilon\left(H_{w}\right)\right)$ which was obtained by Neuberger [3] in the framework of the Overlap formalism [4,5], before the GW relation was rediscovered. Since the Overlap was essentially motivated by the Domain-Wall fermion [6] which was originally apart from the Ginsparg-Wilson fermion, this seems to indicate that the Overlap plays a more fundamental role in resolving the problem of chiral fermions on the lattice than the GW relation. Thus the Overlap possesses the generalized chiral symmetry (1) on the lattice as one of its ingredients. This in turn suggests that any nonperturbative formulation of chiral fermions must comply with the exact chiral symmetry, the GW relation (1).

One of the salient features of any GW Dirac operator satisfying (ii)-(iv) and (4) is that it is not plagued by the notorious problems [7] (e.g., additive mass renormalization, etc.) which usually occur to the Wilson-Dirac lattice fermion operator. However, if one uses the Overlap Dirac operator in lattice QCD, one may encounter the technical problem of taking the square root of a huge positive definite Hermitian matrix $H_{w}^{2}$, which would become quite time-consuming when the condition number of $H_{w}^{2}$ gets too large. Therefore, both in principle and in practice, it is important to understand to what extent one can construct a topologically proper $D$ which satisfies all physical constraints (ii)-(iv), (1) and (2).

The general solution of the Ginsparg-Wilson relation (1) can be formally written as $[9,10]$

$D=D_{c}\left(\mathbb{1}+a R D_{c}\right)^{-1}=\left(\mathbb{1}+a D_{c} R\right)^{-1} D_{c}$,

where $D_{c}$ is any chirally symmetric $\left(D_{c} \gamma_{5}+\gamma_{5} D_{c}=\right.$ 0 ) Dirac operator which must violate at least one of the three properties (ii)-(iv) listed above. Now we require $D_{c}$ to satisfy (iii) and (iv), but violate (ii) (i.e., $D_{c}$ is nonlocal), since (3) can transform the nonlocal $D_{c}$ into a local $D$ on a finite lattice for $R=r \mathbb{I}$ with $r$ in the proper range [11], and also preserves the properties (iii) and (iv).

Then the $\gamma_{5}$-Hermiticity of $D$ (2) is equivalent to the $\gamma_{5}$-Hermiticity of $D_{c}$

$D_{c}^{\dagger}=\gamma_{5} D_{c} \gamma_{5}$,

due to the relation

$D_{c}=D(\mathbb{1}-a R D)^{-1}=(\mathbb{I}-a D R)^{-1} D$,

which is the inverse transformation of (3). Then the chiral symmetry and the $\gamma_{5}$-Hermiticity of $D_{c}$ together implies that $D_{c}$ is antihermitian

$D_{c}^{\dagger}=-D_{c}$,

which is in agreement with the antihermiticity of the massless Dirac operator in continuum. Then there exists one to one correspondence between $D_{c}$ and a unitary operator $V$ such that

$D_{c}=M a^{-1}(\mathbb{1}+V)(\mathbb{1}-V)^{-1}$,

$V=\left(a D_{c}-M\right)\left(a D_{c}+M\right)^{-1}$,

where $M$ is a parameter. Then $V$ also satisfies the $\gamma_{5}$-Hermiticity, $V^{\dagger}=\gamma_{5} V \gamma_{5}$. Substituting (7) into (3), we obtain [9]

$D=M a^{-1}(\mathbb{1}+V)[(\mathbb{1}-V)+M R(\mathbb{1}+V)]^{-1}$.

Note that $D$ is well-defined even if $D_{c}$ has singularities in nontrivial gauge backgrounds. In this case, the index of $D_{c}$ is defined to be the index of $(\mathbb{1}+V)$, the numerator on the r.h.s. of (7). Evidently, the zero modes and the index of $D$ are invariant for any $R$ in 
(3) or $(9)[9,10]$. That is, a zero mode of $D_{c}$ is also a zero mode of $D$ and vice versa, hence,

$$
\begin{aligned}
n_{+}\left(D_{c}\right)= & n_{+}(D), \quad n_{-}\left(D_{c}\right)=n_{-}(D), \\
\operatorname{index}\left(D_{c}\right) & =n_{-}\left(D_{c}\right)-n_{+}\left(D_{c}\right) \\
& =n_{-}(D)-n_{+}(D)=\operatorname{index}(D),
\end{aligned}
$$

where $n_{+}\left(n_{-}\right)$denotes the number of zero modes of $+1(-1)$ chirality.

The conditions (ii)-(iv), (1) and (2) constitute the necessary conditions for $D$ to reproduce the continuum axial anomaly on a finite lattice. For the trivial sector, they are sufficient to guarantee that the correct axial anomaly can be recovered on the lattice.

However, for topologically nontrivial gauge backgrounds, the index as well as the axial anomaly of a lattice Dirac operator $D$ depends on its topological characteristics [12]. If $D$ is topologically proper (i.e., satisfying the Atiyah-Singer index theorem for any gauge background satisfying the topological bound [12]), then the sum of the axial anomaly of $D$ over all sites on a finite lattice is equal to the topological charge of the gauge background. Then it follows that the axial anomaly of $D$ would agree with the topological charge density of the gauge background if $D$ is local.

Now the central problem is how to construct a chirally symmetric and nonlocal $D_{c}$ which satisfies (i), (iii), (iv), and (4). Furthermore we also require that $D_{c}$ is topologically proper. These constitute the necessary requirements [10] for $D_{c}$ to enter (3) such that $D$ could provide a nonperturbative regularization for a massless Dirac fermion interacting with a background gauge field. A systematic construction of $D_{c}$ satisfying (i), (iii), (iv), and (4) has been discussed in Ref. [13]. However, a general prescription for constructing a topologically proper $D_{c}(D)$ still remains an unsolved problem.

Nevertheless, given any lattice Dirac operator $D$, one can always transform $D$ into a GW Dirac operator $D^{\prime}$ which preserves all essential properties of $D$. In other words, if $D$ is topologically proper and satisfies (ii)-(iv) and (2), then the GW Dirac operator $D^{\prime}$ also possesses these properties.

The outline of this paper is as follows. In Section 2 , we define the topologically invariant transformations [15] for lattice Dirac operators. The transformation which can convert any lattice Dirac operator into a GW Dirac operator is derived. In Section 3, we apply this transformation to the Wilson-Dirac lattice fermion operator and obtain a new GW Dirac operator which is local, free of $O(a)$ lattice artifacts and has correct axial anomaly in the trivial sector. In Section 4, we discuss and summarize.

\section{Topologically invariant transformation}

Given any lattice Dirac operator $D$, in general, there are many different ways to extract its chirally symmetric part. For example, one can construct

$D_{s}=\frac{1}{2}\left(D-\gamma_{5} D \gamma_{5}\right)$,

which is chirally symmetric $\left(D_{s} \gamma_{5}+\gamma_{5} D_{s}=0\right)$. However, (12) does not necessarily preserve the property (iii). For example, if one applies this transformation to the Wilson-Dirac operator $D_{W}=\gamma_{\mu} t_{\mu}+$ $W(20)$, one obtains $D_{s}=\gamma_{\mu} t_{\mu}$, the naive fermion operator which suffers from the species doublings. Although $D_{W}$ is free of species doublings in the continuum limit, the transformation (12) cannot preserve this property since $D_{s}$ satisfies (i), (ii) and (iv), thus it must violate (iii) as a consequence of the no-go theorem. Therefore, we need a transformation which preserves the properties (iii), (iv), (2) and (10), but exchanges the locality of $D$ for its chiral symmetry at finite lattice spacing.

Consider the topologically invariant transformation [15] on any $\gamma_{5}$-Hermitian $D$,

$$
\begin{aligned}
\mathscr{T}(R): \quad D & \rightarrow D^{\prime}=\mathscr{T}(R)[D] \equiv D(\mathbb{1}+a R D)^{-1} \\
& =(\mathbb{1}+a D R)^{-1} D,
\end{aligned}
$$

where $R$ is a Hermitian operator which commutes with $\gamma_{5}$ and satisfies $\operatorname{det}(\mathbb{1}+a R D) \neq 0$. Then $D^{\prime}$ is also $\gamma_{5}$-Hermitian.

It is obvious that a zero mode of $D$ is a zero mode of $D^{\prime}$ and vice versa. Suppose $\phi$ is a zero mode of $D$, i.e., $D \phi=0$, then $D^{\prime} \phi=(\mathbb{1}+$ $a D R)^{-1} D \phi=0$. On the other hand, if $\phi$ is a zero mode of $D^{\prime}$, i.e., $D^{\prime} \phi=0$, then we can use the relation $(\mathbb{1}+a D R) D^{\prime}=D$ to obtain $D \phi=0$. Therefore

$D \phi=0 \Leftrightarrow D^{\prime} \phi=0$. 
Moreover, if the zero modes of $D$ have definite chirality, i.e., $D \phi=0$ implies $D \gamma_{5} \phi=0$, then the chirality of $\phi$ is preserved under the transformation (13), i.e., $D^{\prime} \gamma_{5} \phi=0$. In this case, we have

$D \phi_{ \pm}=0 \Leftrightarrow D^{\prime} \phi_{ \pm}=0, \quad n_{ \pm}\left(D^{\prime}\right)=n_{ \pm}(D)$,

$\operatorname{index}\left(D^{\prime}\right)=\operatorname{index}(D)$.

In the trivial sector, $D$ is nonsingular except possibly some 'exceptional' configurations of zero measure, thus (13) is equivalent to

$\mathscr{T}(R): \quad D \rightarrow D^{\prime}$ such that $D^{\prime-1}=D^{-1}+a R$.

Formally, there exists a set of transformations $\{\mathscr{T}(R)\}$ which form an abelian group with group multiplication

$$
\begin{aligned}
\mathscr{T}\left(R_{1}\right) \circ \mathscr{T}\left(R_{2}\right)[D] & =\mathscr{T}\left(R_{1}\right)\left[\mathscr{T}\left(R_{2}\right)[D]\right] \\
& =\mathscr{T}\left(R_{1}+R_{2}\right)[D],
\end{aligned}
$$

where the last equality follows immediately from (14). Explicitly, one checks:

(a) the closure property is satisfied since the sum of any two $\left(\gamma_{5^{-}}\right)$Hermitian operators is $\left(\gamma_{5^{-}}\right)$Hermitian;

(b) the identity element is $\mathscr{T}(0)$;

(c) the inverse of $\mathscr{T}(R)$ is $\mathscr{T}(-R)$;

(d) the associative law

$$
\begin{aligned}
(\mathscr{T} & \left.\left(R_{1}\right) \circ \mathscr{T}\left(R_{2}\right)\right) \circ \mathscr{T}\left(R_{3}\right) \\
& =\mathscr{T}\left(R_{1}\right) \circ\left(\mathscr{T}\left(R_{2}\right) \circ \mathscr{T}\left(R_{3}\right)\right) \\
& =\mathscr{T}\left(R_{1}+R_{2}+R_{3}\right)
\end{aligned}
$$

is also satisfied.

The chiral limit of the transformation (13) is at

$$
R=-\frac{1}{2} a^{-1}\left(D^{-1}+\gamma_{5} D^{-1} \gamma_{5}\right) \equiv R_{c},
$$

which gives the chirally symmetric $D_{c}$

$$
D_{c}=\mathscr{T}\left(R_{c}\right)[D]=2 \gamma_{5} D\left(\gamma_{5} D-D \gamma_{5}\right)^{-1} D \text {. }
$$

Substituting $D_{c}$ (17) into (3), we obtain a GW Dirac operator

$$
D^{\prime}=2 \gamma_{5} D\left(\gamma_{5} D-D \gamma_{5}+2 a D R \gamma_{5} D\right)^{-1} D,
$$

which satisfies the GW relation

$D^{\prime} \gamma_{5}+\gamma_{5} D^{\prime}=2 a D^{\prime} R \gamma_{5} D^{\prime}$ where $R$ is a positive definite Hermitian operator which is local in the position space, commutes with $\gamma_{5}$, and is chosen such that the inverse operator on the r.h.s. of (18) is well-defined. In the following, we shall denote the GW Dirac operator (18) by

$$
\begin{aligned}
D^{\prime} & =\mathscr{T}(R)\left[D_{c}\right]=\mathscr{T}(R) \circ \mathscr{T}\left(R_{c}\right)[D] \\
& =\mathscr{T}\left(R+R_{c}\right)[D] .
\end{aligned}
$$

Note that if $D$ has zero modes in topologically nontrivial gauge backgrounds, then $D_{c}(17)$ has singularities and is not well-defined, however, the GW Dirac operator $D^{\prime}(18)$ is still well-defined. For example, consider the $\gamma_{5}$-hermitian Dirac operator $D=a^{-1}(\mathbb{1}+V)$, where $V$ is a unitary operator having real eigenvalues \pm 1 in nontrivial gauge backgrounds. Then it can be shown that the real $( \pm 1)$ eigenmodes of $V$ have definite chirality and satisfy the chirality sum rule [16], while each complex eigenmode has zero chirality. Thus each +1 eigenmode must be accompanied by a -1 eigenmode of opposite chirality, and vice versa. Now Eq. (17) gives

$D_{c}=2 a^{-1} \frac{\mathbb{1}+V}{\mathbb{1}-V}$,

which has a pole if $D$ has a zero mode. However, Eq. (18) gives a well-defined GW Dirac operator

$$
D^{\prime}=2 a^{-1}(\mathbb{I}+V)[(\mathbb{I}-V)+2 R(\mathbb{I}+V)]^{-1} \text {. }
$$

It is evident that for any two lattice Dirac operators $D^{(1)}$ and $D^{(2)}$ satisfying (2), their corresponding chiral limits obtained from (17), say, $D_{c}^{(1)}$ and $D_{c}^{(2)}$, are in general different. However, they are related by the transformation

$D_{c}^{(1)}=\sum_{i} T_{i} D_{c}^{(2)} T_{i}^{\dagger}$,

where each $T_{i}$ commutes with $\gamma_{5}$. In general, given any two $D_{c}^{(1)}$ and $D_{c}^{(2)}$, it is nontrivial to obtain all $T_{i}$ in (19).

\section{Chirally invariant Wilson-Dirac operator}

The Wilson-Dirac lattice fermion operator [8] can be written as

$D_{W}=\gamma_{\mu} t_{\mu}+W$, 
where

$t_{\mu}(x, y)=\frac{1}{2 a}\left[U_{\mu}(x) \delta_{x+\hat{\mu}, y}-U_{\mu}^{\dagger}(y) \delta_{x-\hat{\mu}, y}\right]$,

$\gamma_{\mu}=\left(\begin{array}{cc}0 & \sigma_{\mu} \\ \sigma_{\mu}^{\dagger} & 0\end{array}\right)$

$\sigma_{\mu} \sigma_{\nu}^{\dagger}+\sigma_{\nu} \sigma_{\mu}^{\dagger}=2 \delta_{\mu \nu}$,

and $W$ is the Wilson term

$$
\begin{aligned}
W(x, y)= & \frac{1}{2 a} \sum_{\mu}\left[2 \delta_{x, y}-U_{\mu}(x) \delta_{x+\hat{\mu}, y}\right. \\
& \left.-U_{\mu}^{\dagger}(y) \delta_{x-\hat{\mu}, y}\right] .
\end{aligned}
$$

The color and Dirac indices have been suppressed in (20). The first term on the r.h.s. of (20) is the naive fermion operator which satisfies properties (i), (ii) and (iv) but violates (iii) since it has $2^{d}-1$ fermion doubled modes. The purpose of the Wilson term is to give each doubled mode a mass of $\sim 1 / a$ such that in the continuum limit each doubled mode becomes infinitely heavy and decouples from the fermion propagator. However, the introduction of the Wilson term has serious drawbacks. It causes $O(a)$ artifacts and also leads to the notorious problems such as additive fermion mass renormalization, vector current renormalization, and mixings between operators in different chiral representations.

The free fermion propagator of $D_{W}$ in momentum space is

$D_{W}^{-1}(p)=\left(\gamma_{\mu} t_{\mu}\right)^{-1} \frac{t^{2}}{w^{2}+t^{2}}+\frac{w}{w^{2}+t^{2}}$,

where $t_{\mu}=i a^{-1} \sin \left(p_{\mu} a\right), t^{2}=a^{-2} \sum_{\mu} \sin ^{2}\left(p_{\mu} a\right)$ and $w=2 a^{-1} \sum_{\mu} \sin ^{2}\left(p_{\mu} a / 2\right)$. We note that in the first term of (25), the doubled modes are decoupled due to the vanishing of the factor $t^{2} /\left(w^{2}+t^{2}\right)$ at the $2^{d}-1$ corners of the Brillouin zone. However, the second term in (25) breaks the chiral symmetry explicitly, and is the source of additive mass renormalization and other notorious problems. Any satisfactory solution to all these problems must get rid of the second term entirely, while keeping the first term intact.
Applying the topologically invariant transformation (17) to $D_{W}$, we obtain the chirally symmetric $D_{c}$,

$D_{c}=\gamma_{\mu} t_{\mu}-W\left(\gamma_{\mu} t_{\mu}\right)^{-1} W$,

which is antihermitian, nonlocal (due to the second term), free of $O(a)$ lattice artifacts, and satisfies (iii), (iv) and (4).

The free fermion propagator of (26) in momentum space is

$D_{c}^{-1}(p)=\left(\gamma_{\mu} t_{\mu}\right)^{-1} \frac{t^{2}}{w^{2}+t^{2}}$,

which is exactly the first term of (25). So, we have 'chiraled away' the second term of (25) through the transformation (17).

Substituting (26) into (3) with $R=r \mathbb{1}$, we obtain a GW Dirac operator

$D=\left[\begin{array}{cc}a r C^{\dagger} C\left(\mathbb{1}+a^{2} r^{2} C^{\dagger} C\right)^{-1} & -C^{\dagger}\left(\mathbb{1}+a^{2} r^{2} C C^{\dagger}\right)^{-1} \\ C\left(\mathbb{1}+a^{2} r^{2} C^{\dagger} C\right)^{-1} & a r C C^{\dagger}\left(\mathbb{1}+a^{2} r^{2} C C^{\dagger}\right)^{-1}\end{array}\right]$,

where

$C=\left(\sigma_{\mu}^{\dagger} t_{\mu}\right)-W\left(\sigma_{\mu} t_{\mu}\right)^{-1} W$.

The locality of $D$ depends on the gauge configuration as well as the value of $r$. If $r$ is zero, then $D$ is equal to $D_{c}$ which is nonlocal, since $D_{c}$ is chirally symmetric, free of species doublings and has correct continuum behavior. For sufficiently smooth gauge configurations, $D$ is local (and not highly peaked in diagonal elements) for $r$ within a proper range. For example, consider the gauge configuration in Fig. 1, $D$ is local for $r \in(0.2,0.8)$. A rigorous proof of the locality of $D$ for a given $r$ and for gauge configurations satisfying a certain bound is beyond the scope of the present paper.

The axial anomaly of $D$ can be written as

$$
\begin{aligned}
\mathscr{A}_{D}(x)= & \operatorname{tr}\left[\left(\mathbb{1}+a^{2} r^{2} C C^{\dagger}\right)^{-1}\right. \\
& \left.-\left(\mathbb{I}+a^{2} r^{2} C^{\dagger} C\right)^{-1}\right](x, x),
\end{aligned}
$$

where tr denotes the trace over the color and spinor space. Since $D_{c}(26)$ in the free fermion limit is free of species doubling and has the correct continuum 


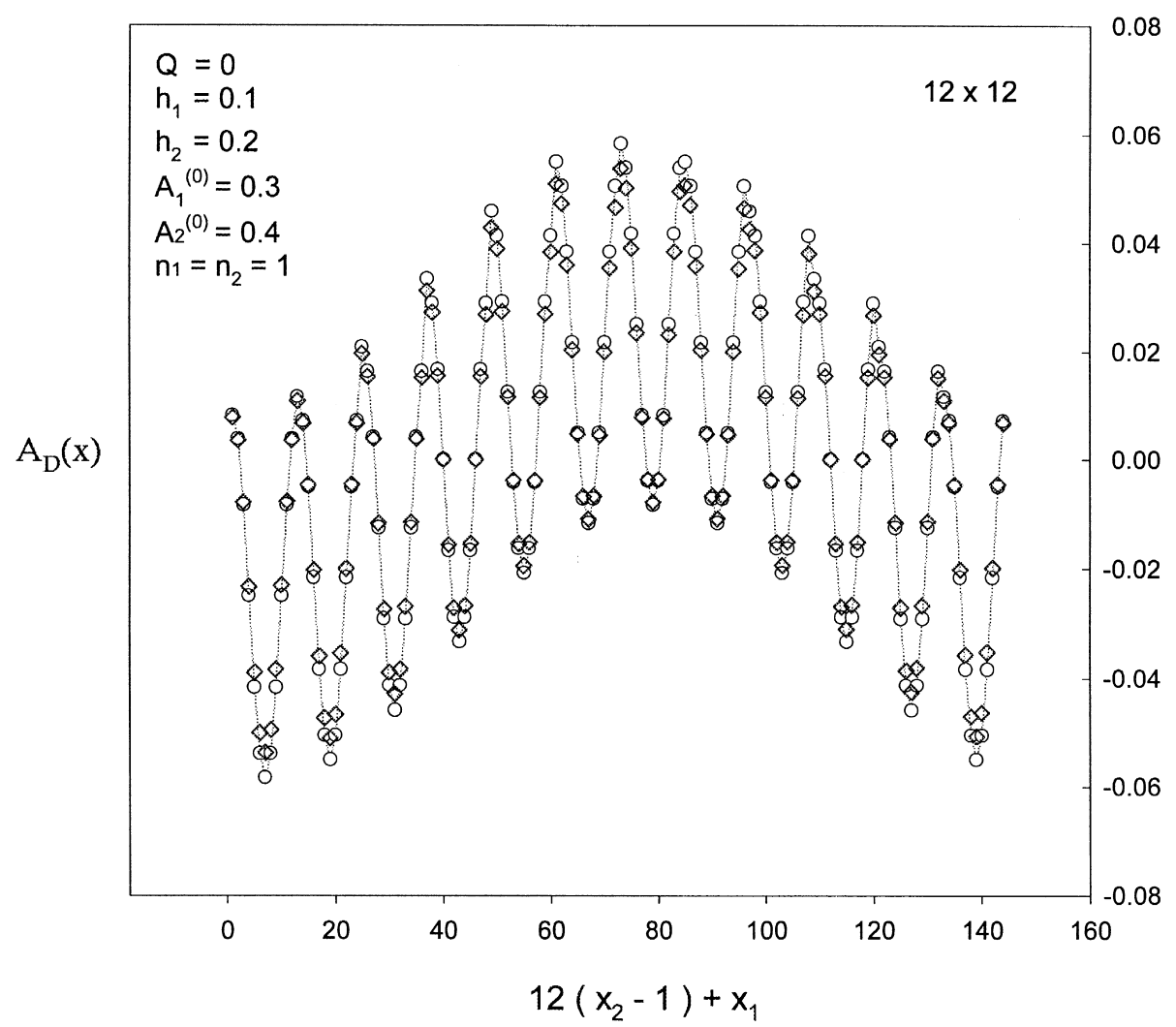

Fig. 1. The axial anomaly $\mathscr{A}_{D}(x)$ [Eq. (30)] of the massless GW Dirac operator $D$ [Eq. (28)] in a trivial gauge background on a $12 \times 12$ lattice. The value of $r$ has been set to 0.5 , and there is no significant changes to $\mathscr{A}_{D}(x)$ for $r \in(0.2,0.8)$. The background gauge field is the same as that in Fig. 1 of Ref. [12]. The axial anomaly $\mathscr{A}_{D}(x)$ is denoted by diamonds. The topological charge density $\frac{1}{2 \pi} F_{12}$ of the gauge background is plotted as circles, which are joined by line segments for the visual purpose.

behavior, the perturbation calculation in Ref. [18] showed that $D(28)$ has the correct axial anomaly in the trivial sector. This has been verified explicitly on finite lattices. An example is shown in Fig. 1, in which the axial anomaly $\mathscr{A}_{D}(x)$ is plotted for each site on a $12 \times 12$ lattice with lattice spacing $a=1$, comparing with the topological charge density $\rho(x)$ $=\frac{1}{2 \pi} F_{12}(x)$ of the trivial gauge background. The position of a site with coordinates $\left(x_{1}, x_{2}\right)$ is represented by an integer $x=12\left(x_{2}-1\right)+x_{1}$, as the $x$-coordinate in Fig. 1 . The axial anomaly $\mathscr{A}_{D}(x)$ is denoted by diamonds, while the topological charge density $\rho(x)$ of the gauge background by circles. The line segments between circles are inserted only for the visual purpose. Evidently $\mathscr{A}_{D}(x)$ agrees with the topological charge density $\rho(x)$ at each site.
It is instructive to compare the axial anomaly of the GW Dirac operator $D$ (28) in a trivial gauge background, as shown in Fig. 1, to that of the Wilson-Dirac operator $D_{W}$ (20) in the same gauge background, as shown in Fig. 1 of Ref. [12]. The former agrees with the topological charge density $\rho(x)$ while the latter does not. This demonstrates that the transformation $\mathscr{T}\left(r+R_{c}\right)$ indeed plays the important role in converting $D_{W}$ into a GW Dirac operator $D=\mathscr{T}\left(r+R_{c}\right)\left[D_{W}\right]$ such that $\mathrm{D}$ is free of $O(a)$ lattice artifacts. Thus $D$ can reproduce the correct axial anomaly even on a finite lattice.

However, this GW Dirac operator (28) does not possess exact zero modes in any topologically nontrivial gauge background (i.e., $D$ is topologically trivial). This is expected since the Wilson-Dirac 
operator $D_{W}$ is topologically trivial, hence the number of zero modes remains zero $\left(n_{ \pm}(D)=n_{ \pm}\left(D_{W}\right)\right.$ $=0)$ under the transformation $D_{W} \rightarrow D=\mathscr{T}(r+$ $\left.R_{c}\right)\left[D_{W}\right]$. Consequently, the sum of the axial anomaly over all sites is zero, not equal to the topological charge of the gauge background. Therefore the axial anomaly of $D$ (or $D_{W}$ ) cannot agree with the topological charge density of the nontrivial gauge background on any finite lattice. It follows that the disagreement must persist even in the continuum limit $a \rightarrow 0$ [12].

Even though the new GW Dirac operator (28) is topologically trivial, it has well-defined chiral properties and is not plaqued by the notorious problems which occur to the standard Wilson-Dirac operator
(20). Therefore, from this viewpoint, it seems that (28) may be an alternative to the Wilson-Dirac operator in lattice QCD, especially for studies involving observables which are dominated by the trivial sector. Further implementations of (28) for lattice QCD are beyond the scope of this paper.

For massive Dirac fermion operator, the mass term $m$ should enter $D$ according to the following transformation [17]

$\mathscr{T}_{m}(R): \quad D_{c} \rightarrow D=\left(D_{c}+m\right)\left(\mathbb{I}+a R D_{c}\right)^{-1}$.

Then for the massive Wilson-Dirac fermion operator, the transformed GW Dirac operator with $R=r \mathbb{1}$ is

$$
D=\left[\begin{array}{cc}
\left(m \mathbb{1}+a r C^{\dagger} C\right)\left(\mathbb{1}+a^{2} r^{2} C^{\dagger} C\right)^{-1} & -(1-m a r) C^{\dagger}\left(\mathbb{1}+a^{2} r^{2} C C^{\dagger}\right)^{-1} \\
(1-m a r) C\left(\mathbb{1}+a^{2} r^{2} C^{\dagger} C\right)^{-1} & \left(m \mathbb{1}+a r C C^{\dagger}\right)\left(\mathbb{1}+a^{2} r^{2} C C^{\dagger}\right)^{-1}
\end{array}\right],
$$

where $C$ is defined in (29).

Although the Wilson-Dirac fermion operator $D_{W}$ is topologically trivial, it can be used to construct the Domain-Wall fermion operator [6] on a five dimensional lattice with fermion mass as a step function in the fifth dimension such that chiral fermions can be realized on the four dimensional walls where the mass defects locate. Then the current flowing into the fifth dimension can induce the correct axial anomaly on the four dimensional walls. Therefore exact zero modes can be reproduced for the chiral fermions on the four dimensional walls for topologically nontrivial gauge backgrounds, and the AtiyahSinger index theorem can be satisfied. However, the chiral symmetry of the fermions residing on the four-dimensional walls is an exact symmetry only in the limit the number of sites in the fifth dimension $N_{s}$ goes to infinity. Thus, at finite $N_{s}$, the DomainWall fermion may suffer from anomalous effects due to chiral symmetry violations [14]. It seems that one may use the new GW Dirac operators (28) and (32) to construct a corresponding Domain-Wall fermion operator. Then it would possess the exact chiral symmetry (1) even at finite $N_{s}$, and the absence of additive quark mass renormalization can be guaranteed.

\section{Summary and discussions}

We can understand the topologically invariant transformation $\mathscr{T}\left(r+R_{c}\right)$ by the following considerations.

Given any lattice Dirac operator $D$, there exists an operator $R$ such that the Ginsparg-Wilson relation (1) can be satisfied. Then, according to (3), there exists a chirally symmetric $D_{c}$ such that $D$ can be written in the form

$D=D_{c}\left(\mathbb{1}+a R D_{c}\right)^{-1}=\mathscr{T}(R)\left[D_{c}\right]$.

Thus the inverse transformation of (33) is

$$
\begin{aligned}
D_{c} & =\mathscr{T}(-R)[D]=D(\mathbb{1}-a R D)^{-1} \\
& \equiv \mathscr{T}\left(R_{c}\right)[D]
\end{aligned}
$$

where

$R_{c}=-R=-\frac{1}{2} a^{-1}\left(D^{-1}+\gamma_{5} D^{-1} \gamma_{5}\right)$.

Once $D_{c}$ is obtained, it can be substituted into (3) with $R$ independent of $D$. In particular, for $R=r \mathbb{1}$, it gives

$$
\begin{aligned}
D^{\prime} & =\mathscr{T}(r)\left[D_{c}\right]=\mathscr{T}(r) \circ \mathscr{T}\left(R_{c}\right)[D] \\
& =\mathscr{T}\left(r+R_{c}\right)[D] .
\end{aligned}
$$


If $D$ is topologically proper and satisfies (iii), (iv) and (2), then these properties are preserved under the transformation (36), i.e., $D^{\prime}$ is topologically proper and satisfies (iii), (iv), (1) and (2). Then it follows that the axial anomaly of $D^{\prime}$ would agree with the topological charge density of the gauge background, provided that $D^{\prime}$ is local (with $r$ in the proper range). On the other hand, if $D$ is topologically trivial, and satisfies (iii), (iv) and (2), then the axial anomaly of a local $D^{\prime}$ (with $r$ in the proper range) would agree with the topological charge density for a trivial gauge background, but not for the nontrivial ones.

For the Wilson-Dirac lattice fermion operator (20), the transformed GW Dirac operator (28) is local, free of $O(a)$ lattice artifacts, has correct axial anomaly in the trivial sector, and is not plagued by the notorious problems (e.g., additive mass renormalization, etc.) which occur to the Wilson-Dirac operator. It seems that (28) or (32) may be an alternative to the Wilson-Dirac operator in lattice QCD.

\section{Acknowledgements}

This work was supported by the National Science Council, ROC under the grant number NSC89-2112M002-017.

\section{References}

[1] H.B. Nielsen, N. Ninomiya, Nucl. Phys. B 185 (1981) 20 [E: B 195 (1982) 541]; B 193 (1981) 173.

[2] P. Ginsparg, K. Wilson, Phys. Rev. D 25 (1982) 2649.

[3] H. Neuberger, Phys. Lett. B 417 (1998) 141; Phys. Lett. B 427 (1998) 353.

[4] R. Narayanan, H. Neuberger, Nucl. Phys. B 443 (1995) 305.

[5] H. Neuberger, Chin. J. Phys. 38 (2000) 533, and references therein; hep-lat/9911022.

[6] D. Kaplan, Phys. Lett. B 288 (1992) 342.

[7] P. Hasenfratz, Nucl. Phys. B 525 (1998) 401.

[8] K. Wilson, in: A. Zichichi (Ed.), New Phenomena in Subnuclear Physics, Plenum Press, New York, 1977.

[9] T.W. Chiu, S.V. Zenkin, Phys. Rev. D 59 (1999) 074501.

[10] T.W. Chiu, Phys. Lett. B 445 (1999) 371.

[11] T.W. Chiu, Phys. Lett. B 474 (2000) 89.

[12] T.W. Chiu, hep-lat/9911010.

[13] T.W. Chiu, Phys. Lett. B 467 (1999) 112.

[14] BC Collaboration, L. Wu R, Nucl. Phys. B (Proc. Suppl.) 83-84 (2000) 224; T. Blum, Domain-Wall fermions, seminars given at the topical program for lattice gauge theories, July 26-30, 1999, NCTS, Hsinchu; N. Christ, Chiral symmetry, lattice QCD and Domain-Wall fermions, talk given at Chiral'99, Sep. 13-18, 1999, Taipei.

[15] T.W. Chiu, hep-lat/9906007.

[16] T.W. Chiu, Phys. Rev. D 58 (1998) 074511.

[17] T.W. Chiu, Phys. Rev. D 60 (1999) 034503.

[18] T.W. Chiu, T.H. Hsieh, hep-lat/9901011. 